Recepción: 21 / 06 / 2018

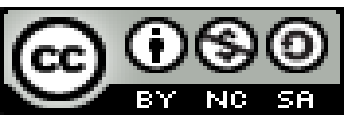

Publicación: 05 / 10 / 2018

\title{
La actitud y motivación de los estudiantes en el logro de aprendizaje de la matemática
}

\section{The attitude and motivation of students in the achievement of learning of mathematics}

\section{A atitude e motivação dos alunos na realização da aprendizagem da matemática}

\author{
Narcisa J. Sánchez Salcán ${ }^{I}$ \\ nsanchez@unach.edu.ec \\ Elena P. Urquizo Cruz II \\ eurquizo@unach.edu.ec
}

Fabián P. Londo Yachambáy ${ }^{\text {III }}$

Correspondencia: nsanchez@unach.edu.ec

\footnotetext{
${ }^{\text {I } M a g i s t e r ~ e n ~ E d u c a c i o ́ n ~ M a t e m a ́ t i c a, ~ D o c t o r a ~ e n ~ E d u c a c i o ́ n, ~ L i c e n c i a d a ~ e n ~ C i e n c i a s ~ d e ~ l a ~ E d u c a c i o ́ n ~ p r o f e s o r a ~ d e ~}$ enseñanza media en la Especialización de ciencias exactas, Docente de la Universidad Nacional de Chimborazo, Riobamba, Ecuador.

II Magister en Educación Superior, Ingeniera Química, Tecnóloga Química Industrial, Docente de la Universidad Nacional de Chimborazo, Riobamba, Ecuador.

${ }^{\text {III }}$ Magister en Gerencia Informática, Ingeniero en Estadística Informática, Docente de la Universidad Nacional de Chimborazo, Riobamba, Ecuador.
} 


\title{
Resumen
}

Los problemas que enfrentan actualmente algunos estudiantes de las instituciones educativas tanto del sector fiscal como privada y de educación superior, son arduos y por diversos razones: los fracasos académicos, obstáculos cognitivos y una serie de problemas que han creado un ambiente negativo en torno a la matemática; buscar alternativas de solución frente a esta problemática se ha convertido en un objetivo para las instituciones educativas; por lo que, el objetivo principal de esta investigación fue diagnosticar las actitudes hacia el logro de aprendizaje en el área de matemática de los y las estudiantes de educación General Básica Superior de las Unidades Educativas fiscales del Distrito Chambo- Riobamba. Se trata de una investigación de tipo descriptiva con un diseño no experimental de tipo correlacional y un muestreo probabilístico de tipo aleatorio estratificado. La técnica utilizada para la recolección de la información fue a través de la aplicación de un cuestionario a la muestra seleccionada, la misma que tuvo validez y fiabilidad. Los resultados indican que, en general, existe una relación estadísticamente significativa entre la actitud y el logro de aprendizaje hacia las matemáticas.

Palabras clave: motivación; rendimiento; matemáticas.

\begin{abstract}
The problems that some students of the educational institutions of the fiscal, private and higher education sectors are currently facing are the low results, academic failures, cognitive obstacles and a series of problems that have created a negative environment around mathematics; finding alternative solutions to this problem has become an objective for educational institutions; Therefore, the main objective of this research was to diagnose the attitudes towards the achievement of learning in the area of mathematics of the students of the General Basic Education of the Fiscal Education Units of the Chambo-Riobamba District. This is a descriptive research with a non-experimental correlational type design and a stratified random type probabilistic sampling. The technique used to collect the information was through the application of a questionnaire to the selected sample, which was valid and reliable. The results indicate that, in general, there is a statistically significant relationship between attitude and achievement of learning towards mathematics.
\end{abstract}

Key words: motivation; performance; mathematics. 


\section{Resumo}

Os problemas enfrentados atualmente por alguns estudantes de instituições de ensino, tanto do sector fiscal e ensino particular e superior, são difíceis e por várias razões: o insucesso escolar, obstáculos cognitivos e uma série de problemas que criaram uma atmosfera negativa em torno da matemático encontrar soluções alternativas para esse problema tornou-se um objetivo para as instituições educacionais; assim, o principal objetivo desta pesquisa foi avaliar atitudes em relação ao aprendizado conquista na área da matemática e estudantes de educação básica geral de imposto mais elevado de Unidades de Educação Riobamba Chambo- Distrito. Trata-se de uma pesquisa descritiva com delineamento de tipo correlacional não experimental e amostragem probabilística estratificada do tipo aleatória. A técnica utilizada para coletar as informações foi através da aplicação de um questionário para a amostra selecionada, que era válida e confiável. Os resultados indicam que, em geral, existe uma relação estatisticamente significante entre atitude e realização de aprendizagem em direção à matemática.

Palavras chave: motivação; performance; matemática.

\section{Introducción}

La Matemática al igual que la Física, la Química y la Biología, contribuyen al adelanto científico de la sociedad, debido a que inciden en casi todas las actividades que desarrolla el hombre, generando una mejor calidad de vida de las personas, instituciones y sociedad en general, siendo por ello pilares fundamentales de la educación a nivel mundial. El aprendizaje de las Ciencias y de manera particular de la Matemática, implica la intervención activa del estudiante durante el proceso, la misma que se desarrolla cuando el estudiante confía en sus capacidades, autoeficacia y muestra compromiso.

Estudios muestran que los resultados de las evaluaciones en matemática a nivel mundial son bajos, por lo que es indispensable apoyar a los estudiantes para que desarrollen habilidades matemáticas y a los docentes para que desarrollen competencias docentes (Caballero, 2016). Existe preocupación de los profesores, por el bajo rendimiento de los estudiantes en el aprendizaje de la matemática, y las consecuencias que tienen en la eficiencia terminal del bachillerato, situación reflejada en las evaluaciones ser Bachiller, donde el área de Matemáticas 
presenta las notas más bajas en comparación con las otras áreas: Ciencias Naturales, Lengua y Literatura y Ciencias Sociales. Según los resultados de las evaluaciones realizadas por el Instituto Nacional de Evaluación Educativa del Ecuador, en donde el área de matemática en el periodo 2016-2017 en la Costa y Sierra en la zona rural presenta una media de 717/1000 puntos, mientras que en la zona urbana se presenta un promedio de 732/1000 puntos, prevaleciendo en ambos casos un nivel de logro Elemental (INEVAL, 2018).

El área de Matemática en el Ecuador según los lineamientos dados por el Ministerio de Educación se encuentra estructurada en tres bloques curriculares: Álgebra y funciones, Geometría y medida, Estadística y probabilidad. A pesar de la forma como se abarca la asignatura tanto en la Educación General Básica y en el Bachillerato, se han generado durante muchos años evidencias claras de actitudes negativas hacia el aprendizaje de las matemáticas, siendo rechazadas y estigmatizadas por los estudiantes producto de factores ambientales y personales, cuya detección constituye una fuente de preocupación para los mismos estudiantes, docentes, padres de familia, especialistas, autoridades educativas y el gobierno en general, por lo que es necesario contrarrestar su influencia negativa con efectividad y así alcanzar los logros de desempeño establecidos por el Ministerio de Educación del Ecuador de manera satisfactoria.

El estudio de estos bloques curriculares en los tres primeros subniveles se trabaja con énfasis en lo concreto y a partir del subnivel superior empieza un tratamiento más abstracto de la Matemática, con la introducción de símbolos y variables; contenidos que se profundizan en el Bachillerato. En donde aparecen problemas hipotéticos, algebraicos, y se busca modelizarlos para su solución (Ministerio de Educación, 2016), del tal manera que el estudiante de sentido práctico a los aprendizajes; situación que en gran medida los docentes no perciben debido al poco interés por la asignatura desde el inicio de la educación básica, y que luego en el Bachillerato al ser la matemática uno de los principales ejes para el desarrollo y aprendizaje de otras ciencias como la Química y la Física, provoca mayor frustración en ellos y el nivel de aprendizaje las demás ciencias básicas.

El estudio de las actitudes positivas y negativas hacia la variedad de aspectos matemáticos actualmente incluidos en el campo de la investigación en la Didáctica Matemática, afirma que las actitudes de los estudiantes influyen en la reprobación de dicha ciencia (Gil, 2005), demostrando 
la influencia que tienen las actitudes en la comprensión del conocimiento y la dimensión sociocognitiva y emocional, reflejado en la preocupación de los estudiantes que no alcanzan el conocimiento de la Matemática, impidiéndoles fructificar en la aplicabilidad de esta área del conocimiento; evidenciándose en los bajos resultados académicos, pruebas estatales, nacionales e internacionales. (Caballero, 2016).

Otro aspecto que profundiza más la problemática es la enseñanza descontextualizada y abstracta, sin considerar los requerimientos e intereses de los estudiantes; además no se ajustan a los nuevos paradigmas constructivistas que impiden el logro de aprendizaje establecido. La dificultad de la resolución de problemas prácticos por parte del estudiante, hace que perciba esta ciencia como algo aburrido; trayendo como resultado que los estudiantes tengan bajo rendimiento académico, que poco a poco se desmotiven, se alejen de la ciencia y pierdan interés en su aplicabilidad. (Rioseco, 2000)

La aplicación de un currículo sobrecargado ha provocado sentimientos de fracaso y baja autoestima en los estudiantes, que se sienten incapaces de asimilar todo aquello que el currículo oficial dice que tendría que aprender, y sentimientos de frustración en el profesorado, que por más que se esfuercen no consigue enseñar todo aquello que se le pide y se le exige que enseñe. Pero las consecuencias negativas van más allá, pues ello contribuye a reforzar la utilización de metodologías de enseñanza expositivas, la misma que es un obstáculo para el diseño y desarrollo de experiencias de innovación pedagógica que dé lugar a un aprendizaje significativo y aporte al perfil de salida del Bachillerato ecuatoriano, puesto que se debe fortalecer la capacidad de razonar, abstraer, analizar, discrepar, decidir, sistematizar y resolver problemas, (Ministerio de Educación 2016).

Por otra parte, en referencia a la operación matemática no tiene por lo general una única vía de cálculo, eso permite que los estudiantes desarrollan también la creatividad cuando hacen cálculos sobre todo mentales pues deben buscar la mejor opción de acuerdo con sus habilidades. (Jiménez 2006), pero debido a su desmotivación no desarrollan su creatividad al momento de resolver problemas matemáticos. 
Por la importancia que tienen las matemáticas dentro del plan de estudios del ministerio de educación y en el desarrollo de los objetivos generales del área de matemática se hace necesario plantearse la siguiente pregunta: ¿De qué manera la actitud se relaciona con el logro de aprendizaje hacia las matemáticas de los estudiantes de educación general básica superior del distrito Chambo -Riobamba?

Las variables de estudio para la presente investigación son: Variable independiente: las actitudes hacia el aprendizaje de la matemática la cual se refiere a la capacidad propia que tienen los seres humanos para enfrentar al mundo y las circunstancias que se les podrían presentar en la vida real. Para Naranjo (como se citó en Gamboa \& Morerira, 2017) indica que las actitudes se adquieren de forma directa e indirecta. El modo directo es cuando la persona las adquiere mediante la experiencia, lo cual le produce satisfacción y significado; por su parte, en el modo indirecto, el individuo adquiere la actitud por medio de otros, a quienes respeta y admira, y que se convierten en modelos auténticos.

La variable dependiente es el logro de aprendizaje en matemática se refiere a las habilidades y conocimientos que el estudiante adquiere o mejora en alguna área específica.

Conjunto de conocimientos, habilidades, destrezas y valores que se pretende que alcance el estudiante en relación con las destrezas con criterio de aprendizaje establecidos en el currículo, que se refleje en los diferentes niveles de la Educación General básica y en el perfil de salida del Bachillerato General Unificado. (Ministerio de Educación, 2016).

\section{Método}

El objetivo de la investigación fue diagnosticar las actitudes hacia el logro de aprendizaje en el área de las matemáticas de los y las estudiantes de educación General Básica Superior de las Unidades Educativas fiscales del Distrito Chambo- Riobamba.

El estudio se realizó a partir de un enfoque de investigación cuantitativa, de carácter descriptivo por el interés de especificar las propiedades, las características y los perfiles importantes de personas, grupos comunidades o cualquier otro fenómeno que se somete a análisis. Se consideró un diseño de la investigación no experimental, de tipo correlacional, el mismo que se utiliza 
cuando se quiere determinar el grado de relación de la variable independiente sobre la variable dependiente. (Ñaupas, 2011).

La población estuvo conformada por los estudiantes de Educación Básica Superior (octavo hasta décimo año) de las unidades Educativas fiscales del distrito Chambo- Riobamba periodo lectivo 2017-2018 con un total de 19159.

Se consideró un muestreo probabilístico de tipo aleatorio estratificado. Este tipo de muestreo, divide a la población en diferentes grupos, con el único fin de darle representatividad a los diversos grupos que son parte de la población en cuestión. (Gómez, 2012, p. 38); es así que se seleccionó a 4 instituciones educativas del distrito Chambo Riobamba, quedando la distribución de la siguiente manera: U.E. Pedro Vicente Maldonado con el 52\%, U.E. Nicanor Larrea con el 14\% U.E. Yaruquies con el 9\%. Y el colegio de Bachillerato Chambo con el 24\%. (Ver tabla 1).

Tabla 1. Muestra

\begin{tabular}{|l|l|l|}
\hline Unidad educativa & Siglas & Muestra \\
\hline Pedro Vicente Maldonado & UEPVM & 198 \\
\hline Nicanor Larrea & UENL & 54 \\
\hline Yaruquies & UEY & 35 \\
\hline Colegio Bachillerato Chambo & CBCH & 90 \\
\hline TOTAL & & 377 \\
\hline
\end{tabular}

La técnica utilizada para la recolección de información en la investigación fue la aplicación de un cuestionario a la muestra antes mencionada. Y para estar seguros que este instrumento responde a las necesidades y expectativas de la investigación y que está de acuerdo con la realidad de la población que se va estudiar, se analizó la fiabilidad a través de un cuestionario piloto, eligiendo aleatoriamente una muestra de 100 entre la población de las unidades educativas del Distrito Chambo Riobamba. Así mismo para encontrar la validez del instrumento se recurrió a la opinión de tres expertos en el área de matemática. 
El cuestionario estuvo constituido por 25 ítems, con los factores: gusto por la matemática, esfuerzo empleado para la matemática, interés por la matemática y la importancia hacia la matemática. Cada ítem de la variable independiente: actitudes hacia la matemática, se evaluó mediante escalas de respuesta: 5 puntos: Totalmente de acuerdo (TA), 4 puntos: De acuerdo (DA), 3 puntos: indeciso (I), 2 puntos: En desacuerdo (D) y 1 punto: Totalmente en desacuerdo (TD). En cambio, para la variable dependiente: logro del aprendizaje fue: 5 puntos: Supera los aprendizajes requeridos (10), 4 puntos: Domina los aprendizajes requeridos (9), 3 puntos: Alcanza los aprendizajes requeridos (7-8), 2 puntos: Está próximo a alcanzar los aprendizajes requeridos (5-6), 1 punto no alcanza los aprendizajes requeridos $(\leq 4)$.

Una vez seleccionada la muestra y elaborado el cuestionario, se procedió a su aplicación en cuatro instituciones educativas del distrito Chambo Riobamba, obviamente con la autorización del director distrital, y con el permiso de las autoridades de cada una de las instituciones educativas; cabe mencionar que cuando se les entregó la encuesta a los estudiantes se les indicó las instrucciones necesarias para el llenado con toda claridad, tratando de motivar la participación e insistiendo en el carácter confidencial de la encuesta.

Finalmente, los datos obtenidos fueron sometidos a un proceso de análisis estadístico con el paquete informático SPSS, tomando en consideración los objetivos de la investigación.

\section{Resultados}

Luego de la aplicación y el procesado de las encuestas a la muestra en estudio se procedió analizar la información de manera descriptiva e inferencial, lo cual permitió realizar las mediciones y comparaciones necesarias para el presente trabajo, cuyos resultados se presentan a continuación:

La fiabilidad del cuestionario a través del coeficiente de alfa de Cronbach fue de 0,964 conforme a la tabla 2, como el coeficiente de confiabilidad hallado se encuentra dentro de la escala 0,72 a 0,99 corresponde a una excelente confiabilidad, (Naupas et al, 2006) con lo que se concluye que el instrumento aplicado es confiable para los fines del presente estudio. 
Tabla 2. Valor del coeficiente alfa de Cronbach

\begin{tabular}{|c|c|}
\hline Alfa de Cronbach & N de elementos \\
\hline, 964 & 5 \\
\hline
\end{tabular}

En la Tabla 3 se muestran los resultados de la aplicación de la prueba de normalidad de los datos, se utilizó la prueba de Kolmogorov Smirnov por contar con una muestra mayor a 50. El nivel de significancia de Z de Kolmogorov Smirnov para las variables medidas es p=0,000 lo cual es inferior a 0,05 por lo que no existe una distribución normal. Por los datos obtenidos de la prueba de normalidad y con la finalidad de evaluar la relación entre las variables en estudio se utilizó la prueba no paramétrica de correlación Rho de Spearman.

Tabla 3. Prueba de normalidad de los datos

\begin{tabular}{|l|r|r|r|}
\hline \multirow{2}{*}{} & \multicolumn{3}{|c|}{ Kolmogorov-Smirnov $^{\mathrm{a}}$} \\
\cline { 2 - 4 } & \multicolumn{1}{|c|}{ Estadístico } & gl & \multicolumn{1}{c|}{ Sig. } \\
\hline Logro_de_aprendizaje &, 236 & 377 &, 000 \\
Actitudes hacia las matemáticas &, 204 & 377 &, 000 \\
\hline
\end{tabular}

\section{Resultados descriptivos de las variables en estudio}

En la tabla $\mathrm{N}^{\circ} 4$ se observa que, de las unidades seleccionadas para la investigación del total de la muestra aplicada (377), 168 estudiantes están indecisos porque no saben si les gusta o no el aprender matemáticas; sin embargo, del mismo grupo se observa que dichos estudiantes no superan los aprendizajes, solamente 145 estudiantes alcanzan los aprendizajes mínimos. 
Tabla $\mathbf{N}^{\mathbf{0}}$ 4. Tabla de contingencia: Gusto por la matemática vs logro de aprendizaje

\begin{tabular}{|c|c|c|c|c|c|c|c|}
\hline & & & \multicolumn{4}{|c|}{ Logro_de_aprendizaje } & \multirow[b]{2}{*}{ Total } \\
\hline & & & No alcanza & $\begin{array}{c}\text { Está } \\
\text { próximo }\end{array}$ & Alcanza & Domina & \\
\hline \multirow[t]{8}{*}{ Gusto } & \multirow{2}{*}{$\begin{array}{l}\text { Totalmente en } \\
\text { desacuerdo }\end{array}$} & Recuento & 33 & 0 & 0 & 0 & 33 \\
\hline & & $\begin{array}{l}\% \text { dentro de } \\
\text { Gusto }\end{array}$ & $100,0 \%$ &, $0 \%$ &, $0 \%$ &, $0 \%$ & $100,0 \%$ \\
\hline & \multirow[t]{2}{*}{ En desacuerdo } & Recuento & 1 & 123 & 8 & 7 & 139 \\
\hline & & $\begin{array}{l}\% \text { dentro de } \\
\text { Gusto }\end{array}$ &, $7 \%$ & $88,5 \%$ & $5,8 \%$ & $5,0 \%$ & $100,0 \%$ \\
\hline & \multirow[t]{2}{*}{ Indeciso } & Recuento & 1 & 5 & 145 & 17 & 168 \\
\hline & & $\begin{array}{l}\% \text { dentro de } \\
\text { Gusto }\end{array}$ &, $6 \%$ & $3,0 \%$ & $86,3 \%$ & $10,1 \%$ & $100,0 \%$ \\
\hline & \multirow[t]{2}{*}{ De acuerdo } & Recuento & 0 & 1 & 3 & 33 & 37 \\
\hline & & $\begin{array}{l}\% \text { dentro de } \\
\text { Gusto }\end{array}$ &, $0 \%$ & $2,7 \%$ & $8,1 \%$ & $89,2 \%$ & $100,0 \%$ \\
\hline \multirow[t]{2}{*}{ Total } & & Recuento & 35 & 129 & 156 & 57 & 377 \\
\hline & & $\begin{array}{l}\% \text { dentro de } \\
\text { Gusto }\end{array}$ & $9,3 \%$ & $34,2 \%$ & $41,4 \%$ & $15,1 \%$ & $100,0 \%$ \\
\hline
\end{tabular}

En la tabla $\mathrm{N}^{\circ} 5$ relacionada al esfuerzo que realizan los estudiantes hacia la matemática se observa que, en las unidades seleccionadas para la investigación del total de la muestra aplicada (377), 135 estudiantes están indecisos en manifestar si realizan un mínimo o un máximo esfuerzo por inmiscuirse en el aprendizaje de la matemática, de este mismo grupo se afirma que 128 estudiantes que corresponde a un porcentaje a 94,8\% alcanza el aprendizaje requerido, no se observa que existan estudiantes que hayan superado los aprendizajes.

Tabla $\mathbf{N}^{\mathrm{a}}$ 5. Tabla de contingencia. Esfuerzo hacia la matemática vs logro de aprendizaje

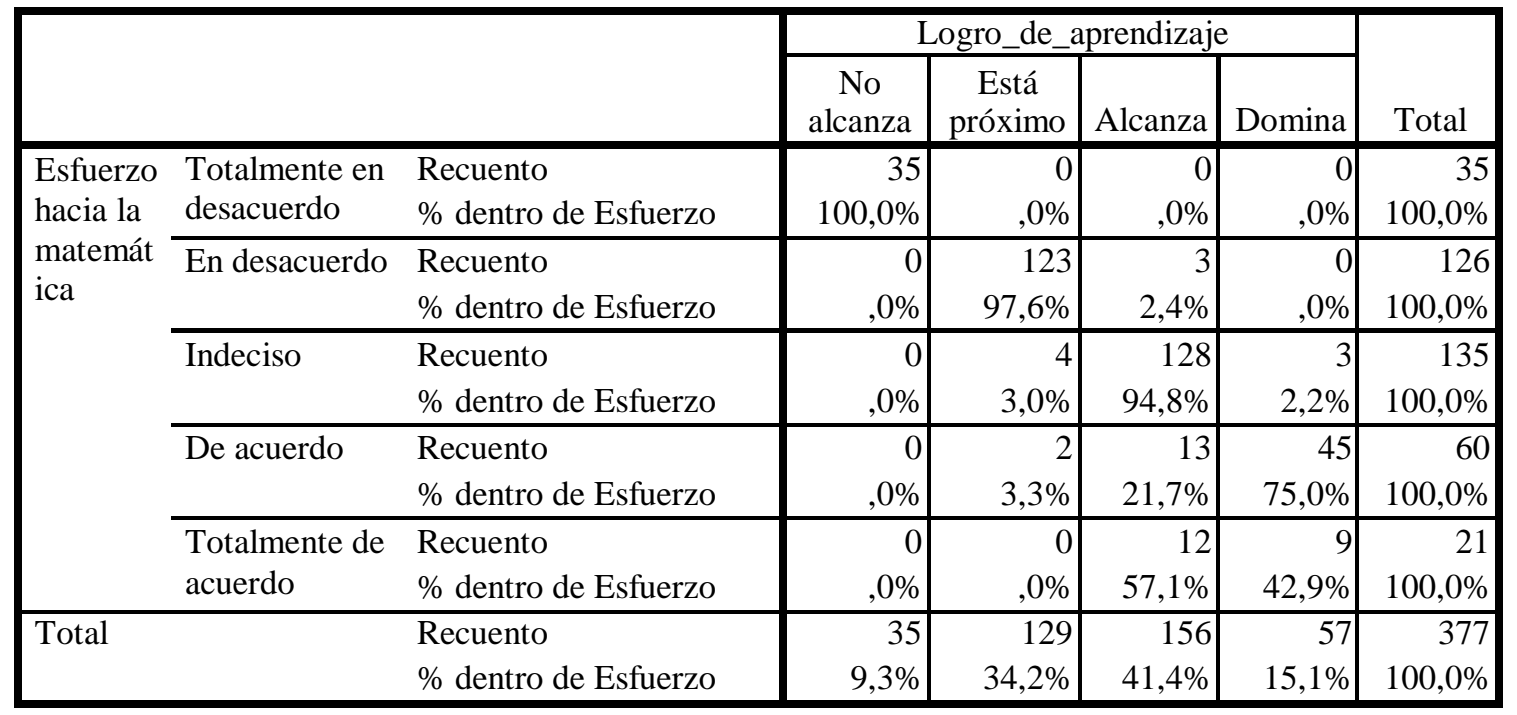


En la tabla $\mathrm{N}^{\circ} 6$ se observa que, en las unidades seleccionadas para la investigación del total de la muestra aplicada (377), 141 estudiantes están indecisos en determinar el interés por aprender matemática, de este mismo grupo de indecisos se observa que casi en su totalidad no alcanzan un máximo estándar de aprendizaje comprendido entre el rango de 9 a 10 puntos.

Tabla N6. Tabla de contingencia: Interés para la matemática vs logro de aprendizaje

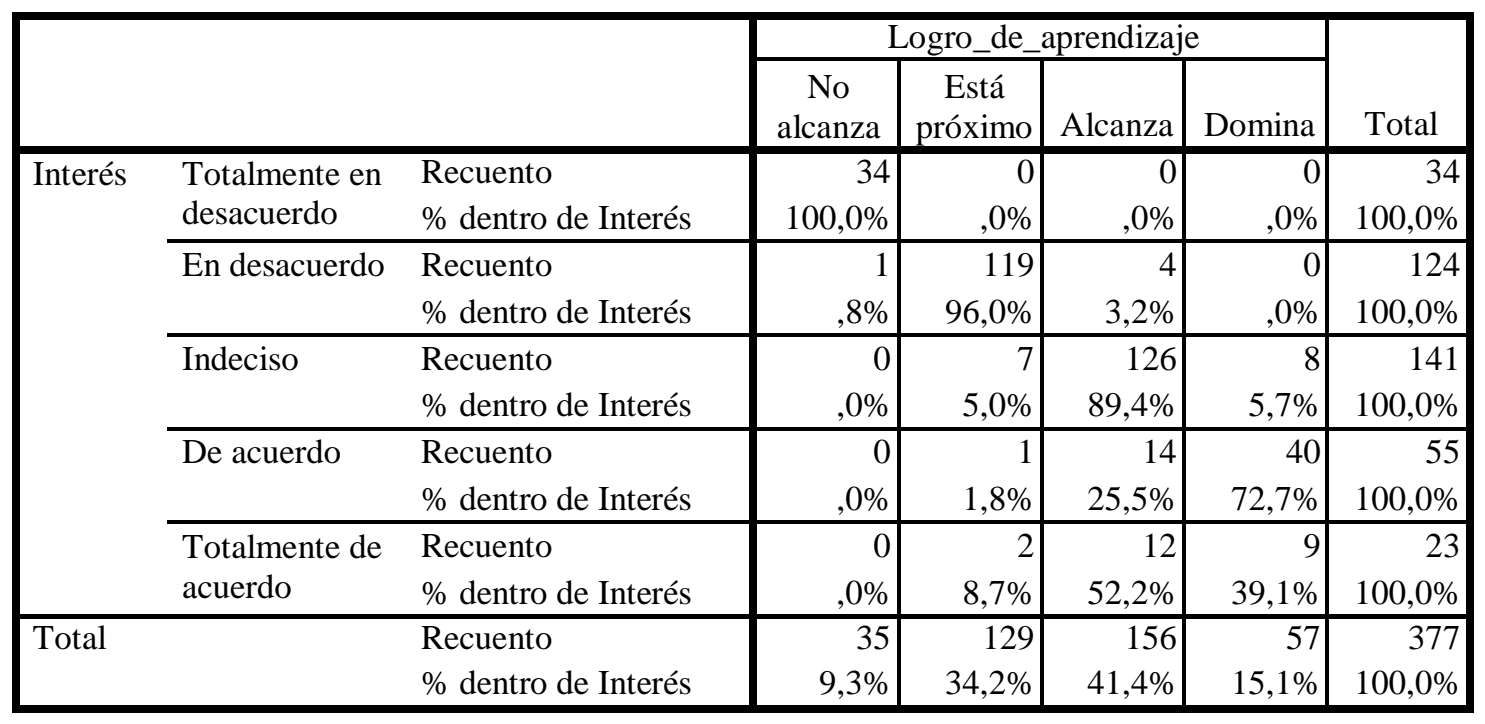

En la tabla $\mathrm{N}^{\mathrm{0}} 7$ se observa que, en las unidades seleccionadas para la investigación del total de la muestra aplicada (377), 134 estudiantes están indecisos en manifestar la importancia hacia la matemática, del mismo grupo 126 estudiantes que corresponde a un porcentaje 94\% alcanza el aprendizaje requerido, no se observa que existan estudiantes que hayan superado los aprendizajes. Lo que se concluye que gran parte de los estudiantes de la localidad consideran a la matemática como una asignatura más, sin darle la importancia debida, de ahí el gran porcentaje de estudiantes indecisos hacia una matemática fundamental para el convivir cotidiano. Por otra parte, la culpa no es solo de los estudiantes sino la responsabilidad también recae en docentes que no desarrollan una metodología apropiada para que los estudiantes sientan el deseo y el entusiasmo por aprender matemáticas, para ello se debe desarrollar políticas que permitan que el docente se capacite en temas pedagógicos y actitudinales hacia la enseñanza de las matemáticas, y de esta forma poder revertir este gran porcentaje de indecisión a un alto índice de apreciación por las matemáticas. 
Tabla No7. Tabla de contingencia: Importancia de la matemática vs logro de aprendizaje

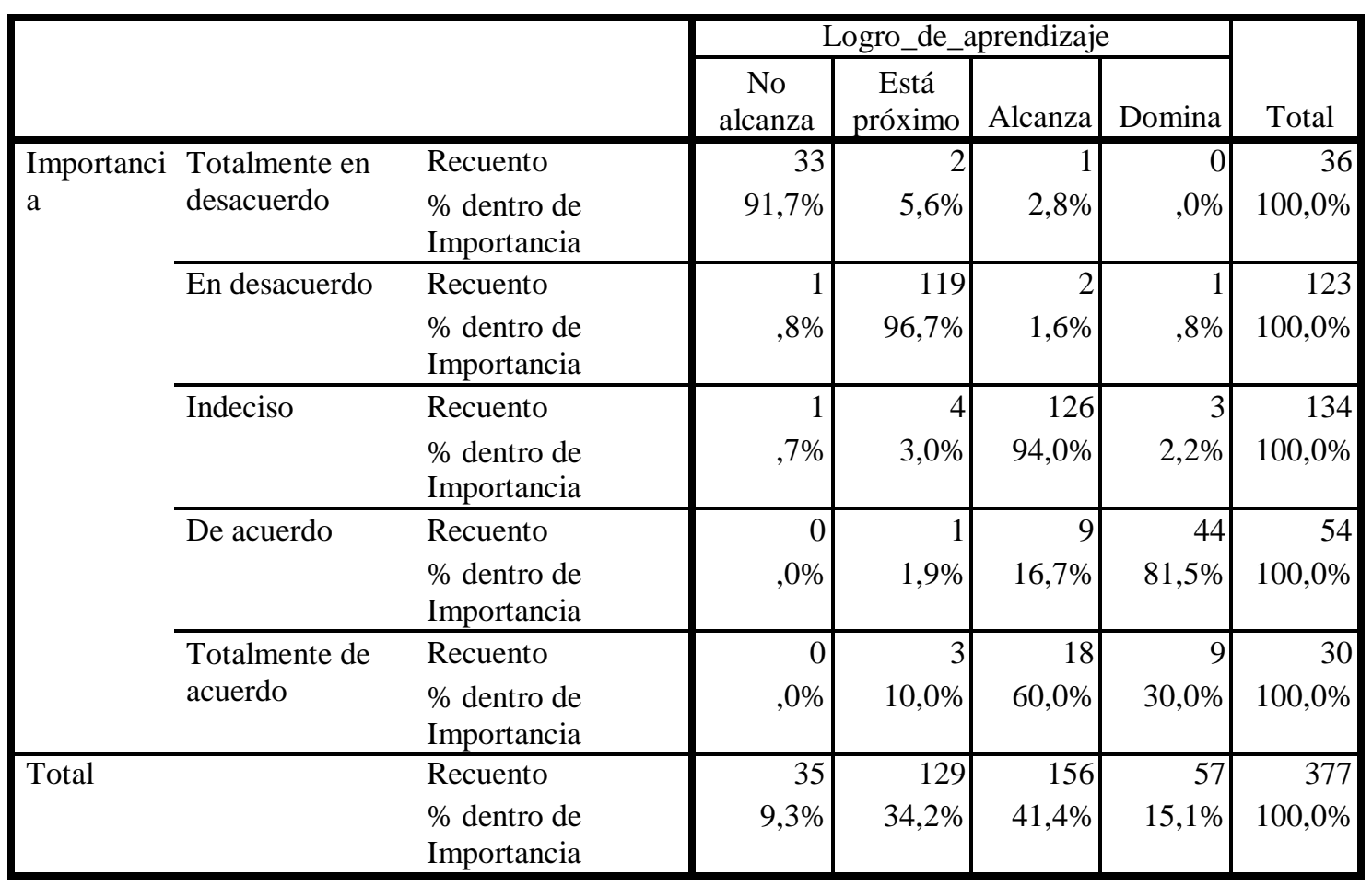

\section{Resultados correlacionales de las variables medidas}

Para evaluar la relación existente entre las variables: Actitudes hacia la matemática y el logro de aprendizaje alcanzado por los estudiantes se empleó el coeficiente de Correlación Rho de Spearman,

De la tabla $\mathrm{N}^{\circ} 8$ se observan la correlación de Spearman entre las variables: actitudes y el logro de aprendizaje hacia la matemática, calculadas al 95\% de confianza y 5\% de significancia. El p valor obtenido es de 0,000 siendo menor al nivel de significancia 0,05, se rechaza la hipótesis nula. Por consiguiente, existe suficiente evidencia estadística para afirmar que: Existe relación directa entre la actitud y el logro de aprendizaje en el área de matemática de los estudiantes de educación básica superior de las unidades educativas del distrito Chambo-Riobamba.

Por otra parte, la prueba estadística Rho de Spearman arroja un valor para r igual a 0,917

para el autor Hernández Sampieri \& Fernández Collado (2010), corresponde a una correlación positiva perfecta esto es, a mayor actitud hacia el aprendizaje de la matemática mayor es el logro de aprendizaje. 
Tabla N 8. Correlación de rho de Spearman entre las variables de estudio

\begin{tabular}{|lll|r|r|}
\hline & & $\begin{array}{c}\text { Competencia } \\
\text { cognitiva }\end{array}$ & actitudes \\
\hline \multirow{2}{*}{ Rho de Spearman } & Logro de aprendizaje & Coeficiente de correlación & 1,000 &, $917^{\text {*** }}$ \\
& & Sig. (bilateral) &, 000 \\
\cline { 2 - 5 } & Actitudes & Coeficiente de correlación &, $917^{* *}$ & 1,000 \\
& & Sig. (bilateral) &, 000 & . \\
\end{tabular}

**. La correlación es significativa al nivel 0,01 (bilateral).

a. $\mathrm{N}$ según lista $=377$

\section{Discusión}

El presente estudio tuvo como propósito diagnosticar las actitudes hacia las matemáticas de los y las estudiantes de educación General Básica Superior del Distrito Chambo- Riobamba, del cuestionario elaborado y aplicado a la muestra en estudio se comprobó que es un instrumento válido y fiable, esto se corrobora con el coeficiente de alfa de Cronbach obtenido $(\alpha=0,964)$, lo cual según los criterio de los autores: Kaplan \& Saccuzzo (1982) manifiestan sobre el valor de fiabilidad para la investigación básica comprendida entre 0.7 y 0.8 ; y en investigación aplicada sobre 0.95; en cambio Loo (como se citó en Frías, 2014) afirma que el valor de consistencia que se considera adecuado es de 0.8 o más; por lo que nuestros resultados estadísticos relativos a la consistencia interna y fiabilidad del cuestionario permiten afirmar que reúne propiedades métricas y adecuadas para adquirir un levantamiento apropiado de información en campo.

De los resultados obtenidos se tiene que existe relación directa entre el factor gusto y el logro de aprendizaje hacia la matemática de los estudiantes de educación básica superior de las unidades educativas del distrito Chambo Riobamba. Además, se observa que los estudiantes con actitudes negativas no alcanzan los aprendizajes requeridos. Por el contrario, los estudiantes con actitudes positivas alcanzan los aprendizajes requeridos provocando en ellos el gusto y confianza hacia la asignatura. Esto coincide con lo manifestado por los autores (Gamboa Araya Ronny \& Moreira Mora Tania, 2017) el gusto que muchos de los estudiantes sienten por las matemáticas se asocia con su rendimiento académico. Si las notas obtenidas por ellos en las actividades de evaluación son altas, se refuerza su confianza en sí mismos con respecto a su desenvolvimiento en la materia y se motivan en su estudio. En caso contrario, la disposición hacia ésta es poco favorable y se genera tristeza, frustración, presión y una serie de pensamiento negativos. 
Por otra parte, existe una relación directa entre el factor interés y el logro de aprendizaje de los estudiantes hacia la matemática, pero con un logro de aprendizaje no tan significativo; esto es, los estudiantes en su mayoría alcanzan los aprendizajes requeridos (7-8 puntos), no existen estudiantes que superen los mismos (10 puntos). Las calificaciones antes mencionadas tendrán como consecuencia que cuando los estudiantes dejen la secundaria con actitudes negativas hacia la matemática, es probable que no tengan la predisposición para seguir aprendiéndola en el futuro, lo que podría tener consecuencias negativas para el desarrollo profesional, entre ellas el fracaso en materias relacionadas con las ciencias exactas, principalmente de la matemática. Por ello, es necesario que los docentes induzcan a los estudiantes a desarrollar una actitud favorable y cognitiva por el gusto, el aprendizaje y estudio de esta ciencia.

También se demuestra que existe relación entre el factor importancia de la matemática con el logro de aprendizaje, con un valor de correlación significativa de 0,88 , lo que implica que las matemáticas son importantes, necesarias y trascendentales para toda persona, considerándose fundamentales en la formación de todo ciudadano. Lo expuesto, se corrobora con la investigación realizada por (Jiménez Bonilla \& Flores López, 2017), quien llega a la conclusión que las actitudes hacia las matemáticas son parte de la formación del estudiantado de Educación Secundaria rural, ya que les permite desarrollar habilidades y destrezas para conocer, valorar, comprender y manejar las emociones y sentimientos de forma armónica en la resolución de situaciones matemáticas vinculadas con la vida cotidiana del estudiantado.

Por consiguiente, se evidencia una relación consecuente y determinista entre las variables: actitud y logro de aprendizaje hacia la matemática, pero con tendencia de niveles medios a niveles bajos, esto conlleva afirmar que desarrollar actitudes positivas hacia la matemática es fundamental, pues el estudiante tendrá una predisposición favorable, se creerá capaz y hará uso de la materia cuando lo sea necesario. Esto coincide con el criterio de los autores (Bazan y Aparicio, 2006) donde manifiestan que mejorar las actitudes implica una serie de actividades que desarrollen las habilidades matemáticas, despierten la curiosidad, estimulen la imaginación del estudiante y ofrezcan oportunidades para el desarrollo de su creatividad. Así mismo, estos resultados son apoyados por los criterios de los autores (Jiménez Bonilla \& Flores López, 2017) donde el objetivo central de la investigación, fue analizar las actitudes hacia las matemáticas del 
estudiantado de Educación Secundaria procedente de una escuela rural, encontrando una actitud globalmente favorable de 82,32. De todo esto se puede desprender que el aprendizaje de las Matemáticas debe estar vinculado fuertemente con la formación de las actitudes positivas hacia el conocimiento, considerando que estas son el factor movilizador al interior de los estudiantes para construir la manera en que estos abordan la ciencia y sus disposiciones generadoras para potenciales transformaciones de los saberes compartidos en las experiencias educativas entre los protagonistas del proceso educativo - estudiantes y profesores.

\section{Conclusiones}

\section{Las conclusiones con las que se llegó al finalizar el presente estudio son las siguientes:}

Los resultados obtenidos de las actitudes hacia el logro de aprendizaje en el área de matemática de los estudiantes de educación básica superior de las unidades educativas del distrito ChamboRiobamba, presentan una tendencia de correlación positiva alta, cuyo valor de correlación es 0.917, la cual es significativa al nivel 0,05 (bilateral).

La investigación ha permitido determinar que los estudiantes de educación básica superior no poseen un criterio de decisión sobre el aprender o no aprender las matemáticas, si les gusta o no les gusta, si les interesa o no les interesa, es decir los estudiantes en gran parte han perdido un total interés sobre el aprendizaje de las matemáticas.

El logro de aprendizaje de los estudiantes no es tan significativo se limitan solamente a la aprobación de la asignatura ( 7 y 8 puntos), no existen estudiantes que se encuentren en la escala de superar los aprendizajes requeridos en la matemática.

Finalmente, como reflexión está en nuestras manos hacer que las matemáticas formen parte de la vida de los estudiantes, motivemos para que tengan la predisposición favorable hacia las matemáticas con intervenciones para mejorar la calidad de los aprendizajes, dejemos de lado aquella época en las que los ejercicios o problemas les causaban temor y tratemos de despertar en los estudiantes el amor por los números. 


\section{Referencias Bibliográficas}

Bazán, J. L., \& Aparicio A. S. (2006). Las actitudes hacia la matemática -estadística dentro de un modelo de aprendizaje. Revista Semestral del departamento de educación, XV, 28, 1-12.

Caballero - Jiménez, F., \& Espínola - Reyna, J. (2016). El rechazo al aprendizaje de las matemáticas a causa de la violencia en el bachillerato tecnológico. Ra Ximhai, 12 (3), 143-161.

Frías, D (2014). Apuntes SPSS. Recuperado de https://www.uv.es/friasnav/ApuntesSPSS.pdf

Gamboa Araya Ronny, \& Moreira Mora Tania Elena. (2017). Actitudes y creencias hacia las matemáticas: un estudio comparativo entre estudiantes y profesores. Revista Actualidades Investigativas En Educación, 1, 1-45.

Gil, N., Blanco, L., y Guerrero, E. (2005). El dominio afectivo en el aprendizaje de las matemáticas. Una revisión de sus descriptores básicos en Revista iberoamericana de educación matemática, Núm. 2, junio, pp. 15-32.

Gómez, S. (2012). Metodología de la investigación. Estado de México.

Hernández, R., Fernández, C., \& Baptista, L. (2010). Metodología de la investigación. México.

Instituto Nacional de Evaluación Educativa INEVAL (2016). Resultados educativos, retos hacia la excelencia. Recuperado de http://www.evaluacion.gob.ec/wpcontent/uploads/downloads/2016/12/CIE_ResultadosEducativos-RetosExcelencia201611301.pdf

Instituto Nacional de Evaluación Educativa. (2018). Análisis de Resultados. Recuperado de http://sure.evaluacion.gob.ec/ineval-dagi-vree-web-2.0-SNAPSHOT/publico/vree.jsf

Jiménez, M., Guerrero E. (2006). Teoría educativa, Secretaría de Educación Pública, Universidad Pedagógica Nacional-Hidalgo, disponible en: http://upnmorelos.edu.mx/2013/documentos_descarga_2013/Antologias_LIE/Tercer_semestre_L IE/teoria_educativa.pdf, 34-67 
Jiménez Bonilla, E., \& Flores López, W. O. (2017). Actitudes hacia las matemáticas: un estudio en una escuela rural de la Costa Caribe Sur de Nicaragua. Revista Universitaria Del Caribe, $18(1), 7$.

Kaplan, Robert M. and Dennis p. Saccuzzo (1982). "Psychological Testing Principles, Applications, and Issues", Monterey, CA: Brooks/Cole Publishing Company.

Ministerio de Educación del Ecuador (2016). Currículo de EGB y BGU. Recuperado de https://educacion.gob.ec/wpcontent/uploads/downloads/2016/03/MATE_COMPLETO.pdf

Ñaupas, H., Mejía, E., Ramírez, E., Villagggómez, A. (2011). Metodología de la Investigación Científica y Asesoramiento de Tesis. Lima., Perú: Centro de Producción Editorial e Imprenta de la Universidad Nacional Mayor de San Marcos.

Rioseco, M. y Romero, R. (2000). "La contextualización de la Enseñanza de la Ciencia como elemento facilitador del aprendizaje significativo". Revista Paideia. Universidad de Concepción.

Universidad de Costa Rica. Instituto de Investigación para el Mejoramiento de la Educación Costarricense., R. G., \& Universidad de Costa Rica. Instituto de Investigación en Educación., T. E. M. (2017). Revista electrónica actualidades investigativas en educación. Actualidades Investigativas en Educación (Vol. 17). 